\title{
Cuantificación práctica del bienestar animal en porcinos de pequeños productores del sur de Veracruz, México
}

\author{
Hanssel Iván Hernández-Antonio1 ${ }^{凶 \mathbb{D}} ;$; Dinora Vázquez-Luna ${ }^{1,2} \otimes \mathbb{0}$; \\ Daniel Alejandro Lara-Rodríguez ${ }^{1,2 *} \otimes(\mathbb{0}) ;$ Marina Martínez-Martínez ${ }^{1} \dot{凶}(\mathbb{D})$
}

\begin{abstract}
${ }^{1}$ Universidad Veracruzana, Facultad de Ingeniería en Sistemas de Producción Agropecuaria, Carretera Costera del Golfo km. 220, Col. Agrícola y Ganadera Michapan. Acayucan, Veracruz, México.

2Universidad Veracruzana, Centro de estudios Interdisciplinarios en Agrobiodiversidad, Carretera Costera del Golfo km. 220, Col. Agrícola y Ganadera Michapan. Acayucan, Veracruz, México.

${ }^{*}$ Correspondencia: dlara@uv.mx
\end{abstract}

Recibido: Julio 2020; Aceptado: Febrero 2021; Publicado: Mayo 2021.

\section{RESUMEN}

Objetivo. Cuantificar el bienestar animal en porcinos de pequeños productores en comunidades del sur de Veracruz. Materiales y métodos. Se realizó un estudio en diez unidades de producción, donde se llevó a cabo un diagnóstico y se analizaron diez variables en los rubros de alimentación, alojamiento, salud y comportamiento de los lechones, de acuerdo con el protocolo Welfare Quality ${ }^{\circledR}$ de la Unión Europea y ponderado de 0 a 1 , donde $0=$ nulo, $0.5=$ regular y $1=$ ideal bienestar animal. Resultados. La relación humano-animal fue satisfactoria; sin embargo, existió bajo confort térmico ligado al tipo de instalaciones. Los principales indicadores de bienestar animal fueron alimentación $0.9 \pm 0.235$, alojamiento $0.6 \pm 0.319$, salud $0.7 \pm 0.252$ y comportamiento $0.9 \pm 0.192$. Conclusiones. La cuantificación del bienestar animal permite identificar de forma práctica aspectos de manejo que los productores han desarrollado de forma intrínseca como la relación humano-animal y la limpieza de las instalaciones. El bienestar animal puede ser cuantificado de forma práctica, ofreciendo alternativas de manejo a los productores, quienes desarrollan actividades adecuadas (limpieza frecuente de las instalaciones) y no adecuadas (alimentación con bajo aporte de proteína, corte de cola y castración).

Palabras clave: Cerdos; manejo del ganado; castración; limpieza; trópico húmedo (Fuente: AGROVOC).

\section{ABSTRACT}

Objective. To quantify the animal welfare of pigs of small producers in communities of southern Veracruz. Materials and methods. We carried out a study in ten production units where we performed a diagnosis and analyzed ten variables in the categories of feeding, housing, health, and behavior of piglets according to the Welfare Quality ${ }^{\circledR}$ protocol of the European Union, which were scored on a 0 to 1 scale, where $0=$ none, $0.5=$ fair, and $1=$ ideal animal welfare. Results. The human-animal 
relationship was satisfactory; however, there was low thermal comfort associated with the type of facilities. The main indicators of animal welfare were feeding $0.9 \pm 0.235$, housing $0.6 \pm 0.319$, health $0.7 \pm 0.252$, and behavior $0.9 \pm 0.192$. Conclusions. The quantification of animal welfare allows the practical identification of management aspects that producers have developed intrinsically, such as the human-animal relationship and cleanliness of the facilities. Animal welfare can be quantified in a practical way, offering management alternatives to the producers, who develop adequate (frequent cleaning of the facilities) and inadequate (low-protein diets, tail docking, and castration) activities.

Keywords: Pigs; livestock management; castration; cleaning; humid tropics (Source: AGROVOC).

\section{INTRODUCCIÓN}

El cerdo doméstico (Sus scrofa domestica) es descendiente del jabalí y es una de las especies más empleadas en la producción animal, desde hace más de 500 años (1). Este ha sido el primer animal que ha permitido a las familias de los pequeños productores, tener recursos mediante la reproducción de lechones, debido a factores como: ciclo biológico corto, alta fecundidad y alimentación omnívora (2). Fue a partir de 1950 que las condiciones de vida impuestas a los cerdos cambiaron hacia una gran transformación zootécnica y tecnológica, donde el manejo restrictivo, así como una convivencia antinatural se convirtieron en el estándar para el alojamiento en naves industriales (3). Actualmente, los sistemas de producción porcícola comerciales se caracterizan por el confinamiento y el manejo intensivo en tecnología y capital, situación que poco ha variado con respecto a décadas anteriores. Lo antes descrito ha afectado aspectos de confort en el reposo, confort térmico y facilidad en el movimiento, teniendo como consecuencia efectos en la salud, el comportamiento y en la productividad de los animales (4), por ello, es importante evaluar el bienestar animal (5). Algunos autores reportan dos tipos de bienestar animal, el físico y el psicológico. El primero lo integran aspectos que pueden ser cuantificados, reparados y están estrechamente relacionados con un buen manejo, mientras que el bienestar psicológico resulta complejo, y hace referencia a la ausencia de miedo al ambiente físico, a las interacciones con seres humanos y al estrés que dichas interacciones puedan ocasionar (6).

El sistema de crianza de ganadería de traspatio no representa un gran gasto económico para las familias que la practican (7), sin embargo deben existir adecuados parámetros productivos y sanitarios basados en el bienestar animal de los sistemas de producción (8). En el sector rural de México, la cría de cerdos, se considera como complementaria del ingreso familiar y el área disponible para su tenencia está en el rango de los 20 y $60 \mathrm{~m}^{2}$, donde el $80 \%$ de los propietarios contribuye de 10 a $30 \%$ del ingreso familiar $(9,10)$, en este caso, el bienestar animal puede quedar en segundo plano, por ello el objetivo del presente estudio fue cuantificar el bienestar animal en porcinos de pequeños productores en comunidades del sur de Veracruz, México.

\section{MATERIALES Y MÉTODOS}

Descripción del área de estudio. La región sur del estado de Veracruz, se caracteriza por su vocación ganadera tradicional (11). El tipo de clima en la zona se sitúa entre el cálido subhúmedo a cálido húmedo. El estudio se desarrolló con productores cooperantes de los municipios de Acayucan 17056'57.48"N , 94'54'53.73"O, Soconusco $17^{\circ} 57^{\prime} 45.71^{\prime \prime} \mathrm{N}, 94^{\circ} 52^{\prime} 51.45^{\prime \prime O}$ y Oluta $17^{\circ} 55^{\prime} 48.27^{\prime \prime} \mathrm{N}, 94^{\circ} 53^{\prime} 46.84^{\prime \prime} \mathrm{O}$ (Figura 1).

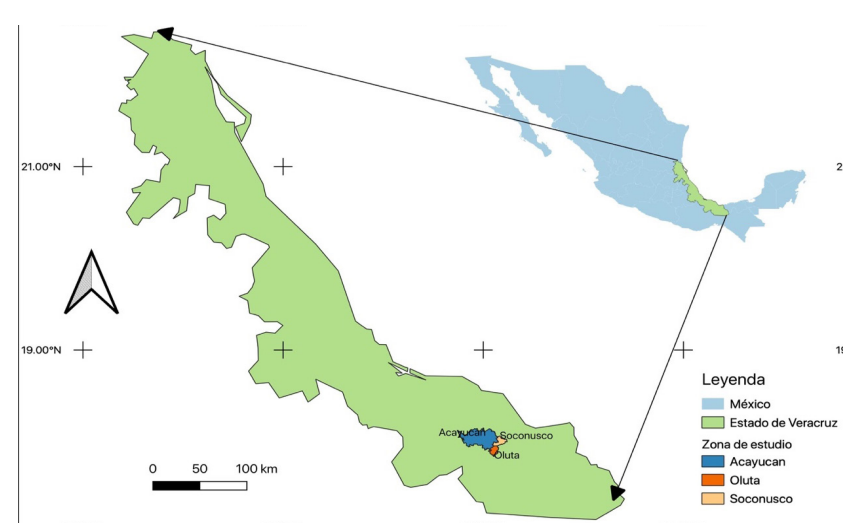

Figura 1. Mapa de localización de productores cooperantes.

Tipo de estudio. Se realizó un estudio trasversal donde se entrevistó a diez productores cooperantes empleando la técnica bola de nieve (12), aplicando un cuestionario estructurado, 
el cual estuvo integrado por las siguientes secciones: alimentación, bienestar animal, comercialización, inventario, parámetros productivos, sanidad, sistemas de explotación, socio cultural. Para el diseño del cuestionario, se revisaron los indicadores de bienestar animal de acuerdo con el protocolo Welfare Quality ${ }^{\circledR}$ de la Unión Europea adaptado para granjeros (13) y se construyó la tabla de indicadores con información de diversos autores (Tabla 1 ).

Se analizaron diez granjas porcícolas de pequeños productores, en donde el mosaico genético es variado, predominando la cruza entre razas Yorkshire y Landrace. En total las granjas contaban con 202 lechones, 99 cerdos en engorda, 237 cerdos destetados, 18 hembras de reemplazo, 20 hembras como pie de cría, así como 14 sementales. Apoyado en una guía de campo, se analizaron las siguientes variables (Tabla 1 ).

\begin{abstract}
Alimentación. En el caso de la ausencia de sed crónica, se basó en la disponibilidad de agua de libre acceso y a voluntad, teniendo en cuenta tanto la calidad como la cantidad, por ello se identificó el número de veces al día que los productores realizaban los recambios de agua en los bebederos de cemento, debido a que en la zona de estudio los recambios son necesarios para mantenerla limpia y fresca. La situación antes descrita es común entre los pequeños productores porcícolas, ya que no cuentan con bebederos automáticos, o válvulas para suministrar el agua a los animales. Cabe mencionar que, en la zona de estudio, la disponibilidad de agua no es un problema. El criterio de ausencia de hambre crónica se midió en función de cuantas veces brindaban alimento y de qué tipo (alimento comercial, frutas o desperdicios).
\end{abstract}

Alojamiento. Con el visto bueno de los productores, se midieron los corrales para identificar si se cumplía con los estándares de alimentación (14) y alojamiento (confort en el descanso, confort térmico y la facilidad en el movimiento), adicionalmente se tomó como variable las dimensiones del espacio recomendado para cerdas, debido a que el bienestar animal de los lechones debería ser medido desde las condiciones de la progenitora, ya que se ha encontrado evidencia de su relación los niveles de cortisol y el crecimiento de lo lechones $(15,16)$. Con respecto al confort térmico, se consideró el estrés por frío, evaluado a partir de la presencia de animales tiritando o apiñados cuando están echados; mientras que el estrés por calor se evaluó a partir de la presencia de animales jadeando (17). La facilidad de movimiento incluyó dos aspectos, animal atado o libre y la posición de los comederos, los cuales de acuerdo con el protocolo Welfare Quality ${ }^{\circledR}$ no deben limitar el movimiento de los porcinos.

Salud. Para la presencia de lesiones, el investigador utilizó una tabla donde identificaba la localización de la lesión por región topográfica y el tipo y el estado de lesión (abierta o curadas). También se tomaron datos de mortalidad, mientras qué para la ausencia de enfermedades y de dolor en las prácticas de manejo (18) se corroboraron los datos de las entrevistas con las visitas de campo, las cuales se realizaron dos veces durante el estudio.

Comportamiento. Para identificar la expresión del comportamiento social se observó a los animales durante 5 minutos, donde también se registraron otras conductas como: Chillidos, problemas de conductas con otros cerdos, mordidas, etc. En la identificación de la relación humano-animal y ausencia de miedo, el investigador estuvo 15 minutos inmóvil observando dentro del corral, donde se registró en una tabla la latencia al primer contacto de los animales con la persona extraña a la unidad de producción y el número total de animales que entraron en contacto con la persona. Además, durante las labores de alimentación y aseo, el investigador observó el comportamiento de los lechones con las personas que realizaban dicho trabajo; la observación se repitió dos veces durante el estudio.

Análisis de datos. Los valores de las tablas fueron convertidos a valores numéricos de acuerdo con los criterios de la Tabla 1, donde 0 correspondió a nulo bienestar animal, 0.5 a regular bienestar animal y 1 al bienestar animal ideal, utilizando las siguientes fórmulas:

Principio $1_{\text {(Alimentación) }}=$ (Ausencia de sed crónica+Ausencia de hambre crónica)/2

Principio $2_{\text {(Alojamiento) }}=$ (Confort en el descanso+Confort térmico+Facilidad en el movimineto) $/ 3$

Principio $3_{\text {(Salud) }}=$ (Lesiones+Ausencia de enfermedades +Dolor por prácticas de manejo)/3

Principio 4 (Comportamiento) $=(E C S+E O C$ + BRHA+Ausencia de miedo)/4 
Hernández-Antonio et al - Cuantificación práctica del bienestar animal en porcinos

Bienestar animal= (Principio $1+$ Principio 2+Principio 3+Principio 4)/4

donde:

ECS $=$ Expresión del comportamiento social

$E O C=$ Expresión de otras conductas

$\mathrm{BRHA}=$ Buena relación humano-animal

El análisis de los datos para las variables numéricas y categóricas se llevó a cabo con el Software IBM SPSS Statistics ${ }^{\circledR}$ para Macintosh, Version 25.0, en donde se analizó la correlación $\mathrm{R}$ de Spearman (19).
Aspectos éticos. La presente investigación fue aprobada por el Consejo Técnico de la Facultad de Ingeniería en Sistemas de Producción Agropecuaria, de la Universidad Veracruzana, en el marco de un trabajo de grado. Las entrevistas, mediciones y demás datos se recabaron al obtener la autorización de los productores. Dada la naturaleza del trabajo que fue de tipo observacional, no fue necesario experimentar con animales.

Tabla 1. Indicadores de bienestar animal basados en el protocolo Welfare Quality ${ }^{\circledR}$ de la Unión Europea (http:// www.welfarequality.net/en-us/home/), modificado de acuerdo con diferentes autores y ponderando a valores, donde: $0=$ nulo bienestar animal, $0.5=$ regular bienestar animal y $1=$ ideal bienestar animal.

\begin{tabular}{|c|c|c|c|c|}
\hline \multirow{2}{*}{ Principio } & \multirow{2}{*}{ Criterio } & \multicolumn{3}{|c|}{ Valor ponderado } \\
\hline & & $\mathbf{0}$ & 0.5 & 1 \\
\hline \multirow{3}{*}{ Alimentación (20) } & Ausencia de sed crónica & 1 recambio de agua/día & 2 recambios de agua/día & 3 recambios de agua/día \\
\hline & & & & \\
\hline & Ausencia de hambre crónica & $1 \mathrm{vez}$ & 2 veces & 3 veces \\
\hline \multirow{4}{*}{ Alojamiento (21) } & Confort en el descanso & $\begin{array}{l}\text { Espacio reducido, } \\
\text { menor a } 2 \mathrm{~m}^{2} / \text { animal }\end{array}$ & $\begin{array}{l}\text { Espacio reducido, } 2 \mathrm{~m}^{2} \\
\text { a } 3 \mathrm{~m}^{2} / \text { animal }\end{array}$ & $\begin{array}{c}\text { Espacio amplio mayor a } \\
3 \mathrm{~m}^{2} / \text { animal, árboles de } \\
\text { sombra }\end{array}$ \\
\hline & Confort térmico & $\begin{array}{c}\text { Estrés por frio: } \\
\text { Animales tiritando } \\
\text { y apiñados al estar } \\
\text { echados. }\end{array}$ & $\begin{array}{c}\text { Estrés por frio: } \\
\text { Animales tiritando } \\
\text { o apiñados al estar } \\
\text { echados. }\end{array}$ & $\begin{array}{c}\text { Estrés por frio: } \\
\text { Ausencia de animales } \\
\text { tiritando o apiñados al } \\
\text { estar echados. }\end{array}$ \\
\hline & comir cetmilco & $\begin{array}{l}\text { Estrés por calor: } \\
\text { Animales jadeando. } \\
\text { Ausencia de sombra. }\end{array}$ & $\begin{array}{c}\text { Estrés por calor: } \\
\text { Animales con jadeo } \\
\text { intermitente. Sombra } \\
\text { insuficiente. }\end{array}$ & $\begin{array}{c}\text { Estrés por calor: } \\
\text { Ausencia de animales } \\
\text { con jadeo. Sombra } \\
\text { adecuada para el total } \\
\text { de la piara. }\end{array}$ \\
\hline & Facilidad en el movimiento & $\begin{array}{c}\text { Animal atado y con } \\
\text { comederos que limiten } \\
\text { el libre paso. }\end{array}$ & $\begin{array}{c}\text { Instalaciones con } \\
\text { comederos que limiten } \\
\text { el libre paso. }\end{array}$ & $\begin{array}{l}\text { Facilidad en el } \\
\text { movimiento }\end{array}$ \\
\hline \multirow{3}{*}{ Salud (22) } & Lesiones & $\begin{array}{c}\text { Presentan lesiones } \\
\text { abiertas }\end{array}$ & $\begin{array}{c}\text { Presentan lesiones } \\
\text { curadas }\end{array}$ & Sin lesiones \\
\hline & Ausencia de enfermedades & $\begin{array}{l}\text { Mortalidad de } 6 \text { al } 10 \% \\
\text { al pre-destete }\end{array}$ & $\begin{array}{l}\text { Mortalidad de } 5 \% \text { al } \\
\text { pre-destete }\end{array}$ & $\begin{array}{c}\text { Mortalidad menor a } 5 \% \\
\text { al pre-destete }\end{array}$ \\
\hline & $\begin{array}{l}\text { Dolor por prácticas de } \\
\text { manejo }\end{array}$ & $\begin{array}{c}\text { Incapacidad física para } \\
\text { desplazarse }\end{array}$ & Dolor o inflamación & Sin presencia de dolor \\
\hline \multirow{4}{*}{ Comportamiento $(23$} & $\begin{array}{l}\text { Expresión del } \\
\text { comportamiento social }\end{array}$ & Bajo & Medio & Alto \\
\hline & $\begin{array}{l}\text { Expresión de otras } \\
\text { conductas }\end{array}$ & Chilla & $\begin{array}{l}\text { Problemas visuales de } \\
\text { otras conductas }\end{array}$ & No presenta \\
\hline & $\begin{array}{l}\text { Buena relación } \\
\text { humano-animal }\end{array}$ & Bajo & Medio & Alto \\
\hline & Ausencia de miedo & Bajo & Medio & Alto \\
\hline
\end{tabular}




\section{RESULTADOS}

Las unidades de producción participantes tuvieron un promedio de dos trabajadores, de los cuales una elevada proporción eran miembros de la familia, el número de vientres en reproducción promedio fue 5.8 con un macho y 1.7 hembras de reemplazo, el número promedio de lechones al destete fue de 14.7, por unidad de producción. El promedio de cerdos destetados por camada, por unidad de producción, fue de 12.1, pesando entre 14 y $25 \mathrm{~kg}$. Los resultados indican que se realizan recambio de agua en los bebederos de tres a seis veces por día y el $100 \%$ de los productores emplea alimento comercial, complementando con desperdicios orgánicos, en algunos casos se les ofrece tortilla (esto no proporciona la proteína requerida por el animal), por ello se obtuvieron valores de 0.7 en ausencia de hambre crónica, ya que esta alimentación no se considera de calidad (Figura 2 ). Se obtuvieron valores promedios de $0.7 \pm 0.252$ con respecto a la salud (Tabla 2), debido a que los productores vacunan, suplementan, bañan y desparasitan. El hecho de realizar la castración tardía o el corte de cola, disminuyeron el principio básico de bienestar de los animales en cuanto a lesiones y dolor por practicas de manejo. Tal como lo muestran los resultados de la correlación $\mathrm{R}$ de Spearman, donde existió una correlación negativa entre las variables de bienestar animal y las variables descole $(-0.824, p=0.002)$ y número de cerdos destinados a la engorda $(-0.803, p=0.005)$, indicando que existe menor bienestar animal al haber mayor numero de cerdos y al desarrollar actividades como el descole.

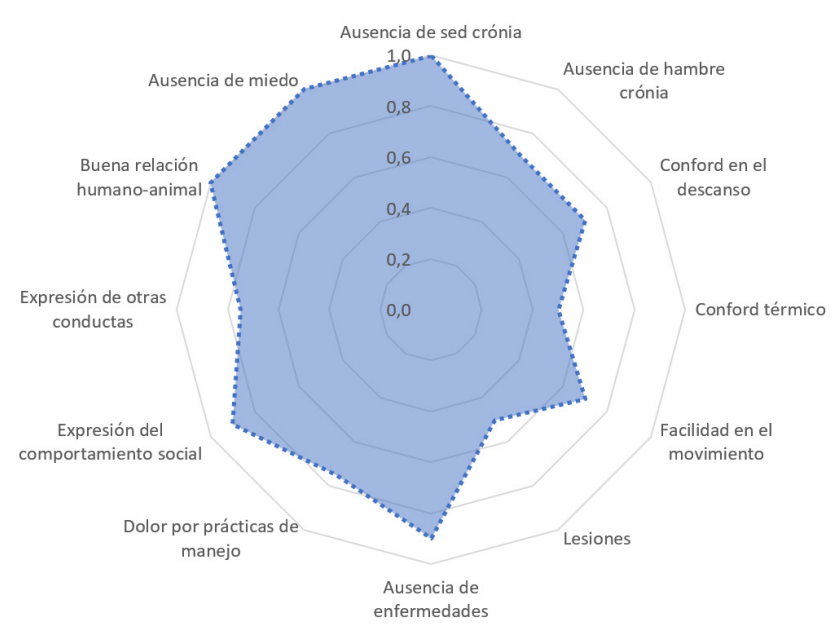

Figura 2. Indicadores de bienestar en lechones de las unidades de producción analizadas.
Tabla 2. Indicadores de bienestar animal de porcinos en comunidades (Acayucan, Oluta y Soconusco) del sur de Veracruz.

\begin{tabular}{cc}
\hline Indicador de bienestar animal & Valores medios \\
\hline Alimentación & $0.9 \pm 0.235$ \\
Alojamiento & $0.6 \pm 0.319$ \\
Salud & $0.7 \pm 0.252$ \\
Comportamiento & $0.9 \pm 0.192$ \\
\hline
\end{tabular}

\section{DISCUSIÓN}

Es importante destacar que en las unidades de producción se realizan prácticas que implican dolor a los animales como son: el corte de cola y la castración, sin la aplicación de anestesia y analgesia, llevándolas a cabo en promedio a los 27 días; pero algunos productores deciden realizar la castración hasta los 41 días, provocando dolor agudo y trauma a los animales. Estos animales traen consigo un comportamiento distinto a los animales castrados en etapas tempranas. Al respecto, de acuerdo con un estudio realizado con consumidores europeos, se encontró que existe la disposición a pagar de 2.449 a $\$ 3.014$ dólares americanos más por salami producido con carne de cerdos castrados con anestesia y analgesia (24). Del mismo modo, estudios recientes indican que el enriquecimiento ambiental combinado con la eliminación de todos los procedimientos invasivos, aumenta el peso al destete en $4.1 \mathrm{~kg}$ e incrementa el peso al sacrificio hasta en $6.3 \mathrm{~kg}$ por cerdo, en comparación con los tratamientos convencionales (25). Por otra parte, el corte de cola esta habitualmente prohibido en Europa, debido a que existen estudios que indican que las tasas de mortalidad pueden ser más altas en los lechones por tener menor talla (34.1\%), en comparación con aquellos que no fueron sometidos al corte de la cola (23\%) (26); pero el manejo depende de las características actitudinales de los productores (27). Con respecto a la legislación Mexicana, existen leyes en algunos Estados de la República que tipifican el maltrato y la crueldad animal, así como la mutilación, sobre todo en animales de compañía (28). Sin embargo, relacionado a la regulación de las prácticas de manejo para las principales especies de interés zootécnico, lo más cercano es la NOM-045-ZOO-1995 donde se establecen las características zoosanitarias para la operación de establecimientos donde se concentren animales para ferias, exposiciones, subastas, tianguis y eventos similares, la cual señala que se deberá procurar el bienestar animal. Del mismo modo, las instalaciones deben ser libres de hambre, de sed y de malnutrición, libre de miedo y estrés sostenidos, libre de incomodidad, libre de dolor, lesión y/o enfermedad, así como libre 
para manifestar un comportamiento natural. Estableciendo algunos criterios con respecto a las condiciones de alojamiento y manejo diferenciado por especie (29).

Con respecto al confort térmico, ciertos productores dejan salir a las cerdas en zonas arboladas, esto puede aumentar el peso al destete a $5.9 \mathrm{~kg}$ por individuo, es decir, 300 g más por animal (30); sin embargo, para los que no realizan este manejo, se sugiere el enriquecimiento ambiental, mediante objetos colgantes y sustrato, disminuyendo el estrés y el comportamiento antisocial, dichas prácticas se han asociado a reducir significativamente los niveles de cortisol (31). Al encontrarse las granjas en clima tropical húmedo o subhúmedo, existen lugares donde hay presencia de árboles frutales, y las temperaturas se encuentran entre 10 y $8^{\circ} \mathrm{C}$ menos, con respecto a lugares sin sombra natural. De igual forma, en facilidad para el movimiento, se promedió un valor de 0.7 , debido a que en ciertas zonas los cerdos son amarrados cercanos a un árbol, resolviendo confort térmico, pero sin facilidad para moverse. Este sistema de producción tiene un componente tradicional, y la toma de decisiones en el sistema está fuertemente condicionada por dichas prácticas (2).

En todos los casos, se encontró una relación humano-cerdo positiva, debido en gran medida a la constante interacción de la familia de los productores, ya que se suele lavar las áreas de manejo, una a dos veces por día, además de ofrecer alimento de manera frecuente. Al respecto, la interacción constante y el uso de la voz humana durante el manejo, disminuyen las reacciones de pánico de los animales hacia los humanos (32), incluso el manejo temprano de los lechones reduce el miedo a las personas, presentando patrones de fuga muy escasos, produciendo cerdos activos y con baja vocalización, reduciendo el estrés y aumentando el peso de los lechones (33). Si bien, se les ofrece a los animales comida en comederos rústicos con forma redonda (mitad de una llanta), es importante considerar que los cerdos de menor rango comen menos que los animales socialmente dominantes (34), por ello, los valores promedio de ausencia de hambre crónica se identificaron con 0.7 , pudiendo existir valores menores al promedio, debido a las condiciones antes descritas.

Finalmente, para el manejo zootécnico adecuado de los porcinos, es necesario mejorar sus condiciones de bienestar animal tanto en las cerdas como en las crías (35). Una estrategia es permitir que las cerdas críen a los lechones sin confinar a los animales (30). Dicho conocimiento es aplicado por parte de los productores, debido en gran medida, a sus costumbres. Por ello, su evaluación en el campo del bienestar animal es una herramienta que coadyuva a mejorar la producción de forma sostenible, aunque para lograr implementarlos es necesario establecer relaciones humano-animal positivas, teniendo en cuenta los criterios previamente definidos, entre dichos criterios destacan que los cerdos no sean maltratados durante el manejo, tengan la sanidad necesaria para no presentar lesiones o enfermedades y otras anormalidades conductuales (chillidos) que afecten la productividad de los mismos (36).

En conclusión, la cuantificación del bienestar animal permite identificar de forma práctica aspectos de manejo que los productores han desarrollado de forma intrínseca como la relación humano-animal y la limpieza de las instalaciones; pero existen otros que afectan severamente el bienestar animal como son: el corte de cola y la castración en animales a edades inadecuadas, así como la alimentación con residuos de comida con poco valor proteico.

\section{Conflictos de interés}

Los autores declaran que no existe conflicto de interés.

\section{Agradecimientos}

Los autores agradecen al CONACyT, en especial al Sistema Nacional de Investigadores por el apoyo económico para la presente publicación.

\section{REFERENCIAS}

1. Hlongwane NL, Hadebe K, Soma $P$, Dzomba EF, Muchadeyi FC. Genome Wide Assessment of Genetic Variation and Population Distinctiveness of the Pig Family in South Africa. Front Genet. 2020; 11:344. https://doi.org/10.3389/fgene.2020.00344
2. Martínez-Castañeda FE, Perea-Peña M. Estrategias locales y de gestión para la porcicultura doméstica en localidades periurbanas del Valle de México. Agric Soc Desarro. 2012; 9(4):411-425. https://www. revista-asyd. $\mathrm{mx} / \mathrm{index} . \mathrm{php} / \mathrm{asyd} / \mathrm{article/}$ view/1180/515 
3. Temple D, Courboulay V, Velarde A, Dalmau $A$, Manteca $X$. The welfare of growing pigs in five different production systems in France and Spain: assessment of health. Anim Welfare. 2012; 21(2):257-271. https://doi. org/10.7120/09627286.21.2.257

4. Mora QRA. Enfoque eficiente del bienestar animal en el contexto nacional e internacional. Rev Colomb Cienc Pecu. 2011; 24(3):327331. https://revistas.udea.edu.co/index. $\mathrm{php/rccp/article/view/324691/20782035}$

5. Kittawornrat A, Zimmerman JJ. Toward a better understanding of pig behavior and pig welfare. Anim Health Res Rev. 2011; 12(1):25-32. https://doi.org/10.1017/ $\underline{\mathrm{S} 1466252310000174}$

6. Johnson N, Iorliam B. Minimizing Stress In Pigs In Confinements When Conducting Research-A Review. Int J Adv Res Publ. 2020; 4(3):158-160. http://www.ijarp. org/published-research-papers/mar2020/ Minimizing-Stress-In-Pigs-In-ConfinementsWhen-Conducting-Research-A-Review.pdf

7. Gutiérrez-Ruiz E, Aranda-Cirerol F, RodríguezVivas R, Bolio-González M, RamírezGonzález S, Estrella-Tec J. Factores sociales de la crianza de animales de traspatio en Yucatán, México. Bioagrociencias. 2012; 5:20-28. https://www.ccba.uady. $\mathrm{mx/}$ bioagro/V5N1/V5N1.pdf

8. Benítez-Meza A, Gómez-Gurrola A, Hernández-Ballesteros J, NavarreteMéndez R, Moreno-Flores L. Evaluación de parámetros productivos y económicos en la alimentación de porcinos en engorda. Aban Vet. 2015; 5(3):36-41. https:// abanicoacademico.mx/revistasabanico/ index.php/abanico-veterinario/article/ view/80/63

9. Sanvicente L, Vargas L, Bustamante G, Jaramillo V. La crianza de cerdos en vida libre y pecaríes silvestres en zonas de transición de áreas protegidas del sureste de México. Arch Zootec. 2020; 69(266):216-224. https://doi.org/10.21071/az.v69i266.5117

10. González OF, Pérez MA, Ocampo FI, Paredes SJA, de la Rosa PP. Contribuciones de la producción en traspatio a los grupos domésticos campesinos. Estud Soc. 2014; 22(44):146-170. https://www.ciad. $\mathrm{mx/}$ estudiosociales/index.php/es/article/ view/145/92
11. Reyna-Ramírez CA, Fuentes-Ponce $\mathrm{MH}$, Rossing WA, López-Ridaura $\mathrm{S}$. Caracterización de unidades de producción familiar agropecuarias mesoamericanas. Agrociencia. 2020; 54(2):259-277. https://agrociencia-colpos.mx/index.php/ agrociencia/article/view/1905/1902

12. Stockemer D. Conducting a Survey. In: Stockemer D, editor. Quantitative Methods for the Social Sciences: A Practical Introduction with Examples in SPSS and Stata. Cham: Springer Inte Publ; 2019.. https://doi. org/10.1007/978-3-319-99118-4 5

13. Courboulay V, Meunier-Salaün M-C, Stankowiak M, Pol F. BEEP: An advisory pig welfare assessment tool developed by farmers for farmers. Lives Sci. 2020; 240:104107. https://doi.org/10.1016/j. livsci.2020.104107

14. Hawe SJ, Scollan N, Gordon A, Muns $R$, Magowan $E$. Impact of feeding low and average birthweight pigs on a weight basis post-weaning on growth performance and body composition. Livest Sci. 2020;241:104233. https://doi. org/10.1016/j.livsci.2020.104233

15. Verdon $\mathrm{M}$, Hansen $\mathrm{CF}$, Rault JL, Jongman $\mathrm{E}$, Hansen LU, Plush $\mathrm{K}$, et al. Effects of group housing on sow welfare: A review. J Anim Sci. 2015; 93(5):1999-2017. https://doi. org/10.2527/jas.2014-8742

16. Hemsworth P. Key determinants of pig welfare: implications of animal management and housing design on livestock welfare. Anim Prod Sci. 2018; 58(8):1375-1386. https://doi.org/10.1071/AN17897

17. Manteca X, Mainau D, Temple D. Bienestar animal. Manual de Buenas Prácticas de Producción Porcina Lineamientos generales para el pequeño y mediano productor de cerdos Red Porcina Iberoamericana. 2012: 97-111. http://200.23.35.7/ archivos/productos/vinculacion/ librosdivulgacion/300700005 1.pdf

18. Varón-Álvarez L, Romero M, Sánchez J. Caracterización de las contusiones cutáneas e identificación de factores de riesgo durante el manejo presacrificio de cerdos comerciales. Arch Med Vet. 2014; 46(1): 93-101. https://doi.org/10.4067/ $\underline{\text { S0301-732X2014000100013 }}$ 
19. Verma J. Data analysis in management with SPSS software: Springer Sci Bus Media; 2012. https://www.springer.com/ $\mathrm{gp} /$ book/9788132207856

20. García-Contreras A, Ortega YDL, Yagüe A, González JG, Artiga CG. Alimentación práctica del cerdo. Rev Complut Cienc Vet. 2012; 6(1):21. http://dx.doi.org/10.5209/ rev RCCV.2012.v6.n1.38718

21. Hemsworth PH, Rice M, Nash J, Giri K, Butler KL, Tilbrook AJ, et al. Effects of group size and floor space allowance on grouped sows: Aggression, stress, skin injuries, and reproductive performance1. J Anim Sci. 2013 2013; 91(10):4953-4964. https://doi. org/10.2527/jas.2012-5807

22. Cadéro A, Aubry A, Dourmad JY, Salaün Y, Garcia-Launay F. Effects of interactions between feeding practices, animal health and farm infrastructure on technical, economic and environmental performances of a pigfattening unit. animal. 2020: 1-12. https:// doi.org/10.1017/S1751731120000300

23. Vitali $M$, Santacroce $E$, Correa F, Salvarani $C$, Maramotti FP, Padalino B, et al. On-Farm Welfare Assessment Protocol for Suckling Piglets: A Pilot Study. Animals. 2020; 10(6):1016. https://doi.org/10.3390/ani10061016

24. Heid A, Hamm U. Animal welfare versus food quality: Factors influencing organic consumers' preferences for alternatives to piglet castration without anaesthesia. Meat Sci. 2013; 95(2):203-211. https://doi. org/10.1016/j.meatsci.2013.04.052

25. Morgan L, Itin-Shwartz B, Koren L, Meyer JS, Matas D, Younis A, et al. Physiological and economic benefits of abandoning invasive surgical procedures and enhancing animal welfare in swine production. Sci Rep. 2019; 9(1):16093. https://doi.org/10.1038/ s41598-019-52677-6

26. Van Beirendonck S, Driessen B, Verbeke G, Permentier L, Van de Perre V, Geers R. Improving survival, growth rate, and animal welfare in piglets by avoiding teeth shortening and tail docking. J Vet Behav. 2012; 7(2):88-93. https://doi. org/10.1016/j.jveb.2011.08.005

27. Kauppinen T, Vesala KM, Valros A. Farmer attitude toward improvement of animal welfare is correlated with piglet production parameters. Livest Sci. 2012; 143(2):142-150. https://doi.org/10.1016/j. livsci.2011.09.011
28. Garcia ADM. Tipificación del maltrato animal en el Estado de Hidalgo, México: Derecho Animal Forum of Animal Law Studies; 2016.

29. Vargas-Bello-Pérez $E$, Miranda-de la Lama GC, Teixeira DL, Enríquez-Hidalgo D, Tadich $\mathrm{T}$, Lensink J. Farm Animal Welfare Influences on Markets and Consumer Attitudes in Latin America: The Cases of Mexico, Chile and Brazil. J Agric Environ Ethics. 2017; 30(5):697-713. https://doi.org/10.1007/ s10806-017-9695-2

30. Nowland TL, van Wettere WHEJ, Plush KJ. Allowing sows to farrow unconfined has positive implications for sow and piglet welfare. Applied Anim Behav Sci. 2019; 221:104872. https://doi.org/10.1016/j. applanim.2019.104872

31. Yang $\mathrm{CH}$, Ko HL, Salazar LC, Llonch $\mathrm{L}$, Manteca X, Camerlink I, et al. Pre-weaning environmental enrichment increases piglets' object play behaviour on a large scale commercial pig farm. Appl Anim Behav Sci. 2018; 202:7-12. https://doi.org/10.1016/j. applanim.2018.02.004

32. Bensoussan $S$, Tigeot $R$, Meunier-Salaün $\mathrm{M}-\mathrm{C}$, Tallet $\mathrm{C}$. Broadcasting human voice to piglets (Sus scrofa domestica) modifies their behavioural reaction to human presence in the home pen and in arena tests. Appl Anim Behav Sci. 2020; 225:104965. https://doi. org/10.1016/j.applanim.2020.104965

33. de Oliveira D, Paranhos da Costa MJR, Zupan M, Rehn T, Keeling LJ. Early human handling in non-weaned piglets: Effects on behaviour and body weight. Appl Anim Behav Sci. 2015; 164:56-63. https://doi. org/10.1016/j.applanim.2015.01.002

34. Fraser D, Duncan IJH, Edwards SA, Grandin T, Gregory NG, Guyonnet V, et al. General Principles for the welfare of animals in production systems: The underlying science and its application. Vet J. 2013; 198(1):19-27. https://doi.org/10.1016/j.tvjl.2013.06.028

35. Nowak B, Mucha A, Kruszyński W, Moska M. Phenotypic correlations between reproductive charac-teristics related to litter and reproductive cycle length in sows. Czech J Anim Sci. 2020; 65(6):205-212. https:// doi.org/10.17221/108/2020-CJAS

36. Manteca X. Bienestar animal en explotaciones de porcino. Revista Colombiana de Ciencias Pecuarias. 2011; 24(3):303-305. https:// revistas.udea.edu.co/index.php/rccp/ article/view/324687/20782025 